\title{
Transverse impedance of a smooth flat taper
}

\author{
Samuel Krinsky \\ Brookhaven National Laboratory, Upton, New York 11973, USA
}

(Received 31 August 2005; published 27 December 2005)

\begin{abstract}
Stupakov has used a perturbation method to estimate the transverse impedance at zero frequency of a rectangular collimator having characteristic taper length $\ell$, half-width $w$, and average vertical halfaperture $b_{0}$, under the condition $b_{0} \ll w \ll \ell$. We use the boundary perturbation method to approximate the transverse impedance of a flat, slowly tapered chamber in the complementary limit, $w=\infty$.
\end{abstract}

DOI: 10.1103/PhysRevSTAB.8.124403

PACS numbers: 41.20.Jb, 41.20.-q, 41.60. $-\mathrm{m}$

\section{INTRODUCTION}

Small gap undulators are a key element in the design of modern high brightness synchrotron light sources. An important design issue is the determination of the transverse impedance of the tapered vacuum chambers for these devices [1]. The geometric impedance of a slowly tapered structure with circular cross section has been discussed by Yokoya [2]. Further understanding was provided by Stupakov [3] who showed that Yokoya's results were valid down to zero frequency. Stupakov [4,5] then presented an analysis of a flat tapered structure, and found that the impedance at low frequency can be significantly larger than that of a chamber with circular cross section.

In order to study the transition between circular and flat geometry, Podobedov and Krinsky [6] recently considered the case of a tapered chamber with elliptical cross section.

Yokoya's [2] result for the low frequency transverse impedance of an axially symmetric tapered transition is given by

$$
Z_{\perp}^{\text {round }}(k) \cong \frac{j Z_{0}}{2 \pi} \int_{-\infty}^{\infty} d z \frac{a^{\prime}(z)^{2}}{a(z)^{2}},
$$

where $k$ is the wave number of the perturbing field, $Z_{0}$ is the free space impedance, $a(z)$ is the radius of the tapered chamber, and the prime denotes derivative with respect to the axial coordinate $z$. If the variation of the radius takes place over a characteristic distance $\ell$, then Yokoya's approximation holds under the conditions $a_{0} \ll \ell$ and $k a_{0}^{2} \ll$ $\ell$, where $a_{0}$ is the average radius.

Stupakov [4] has derived an approximation for the vertical impedance of a flat rectangular chamber of constant half-width $w$ and varying half-gap $b(z)$,

$$
Z_{y}^{\text {flat }}(k) \cong \frac{j Z_{0} w}{2} \int_{-\infty}^{\infty} d z \frac{b^{\prime}(z)^{2}}{b(z)^{3}}
$$

If the variation of the gap takes place over a distance $\ell$, then Stupakov's approximation can be expected to hold under the conditions $b_{0} \ll w \ll \ell$ and $k w^{2} \ll \ell$, where $b_{0}$ is the average half-gap. The impedance of the flat chamber (1.2) has a form similar to Eq. (1.1) for a circular chamber, but is larger by a factor $\sim \pi w / b_{0}$. Within this approximation the impedance increases linearly with the chamber width. It is intuitively clear that this linear rise cannot continue forever, and one expects that the impedance will reach a saturation value once the width is large compared to the axial length scale $\ell$. This expected behavior is not exhibited within Stupakov's [4] first-order perturbation theory.

In order to achieve more insight into this problem, we use the boundary perturbation method [7-10] to derive an analytic approximation for the transverse impedance of a flat tapered chamber of infinite width and slowly varying vertical aperture. Our calculation is an extension from circular to flat geometry of the approach developed by Cooper, Krinsky, and Morton [9].

\section{CIRCULAR CHAMBER}

Let us review the results obtained for the transverse impedance of an axially symmetric chamber with slowly varying radius. The fractional deviation of the radius from its average value $a_{0}$ is denoted by $S(z)$ and is expressed as a Fourier integral

$$
S(z) \equiv \frac{a(z)-a_{0}}{a_{0}}=\int_{-\infty}^{\infty} d q A(q) e^{j q z} .
$$

The approaches of Yokoya [2] and Stupakov [3-5] require only that the derivative $S^{\prime}(z)$ be small, whereas the boundary perturbation method [7-9] requires the additional restriction that $S(z)$ also be small.

The work presented in Refs. [8,9] was carried out for a structure that is periodic in the axial direction. Yokoya [2] took the long period limit to obtain the impedance for a nonperiodic structure and found

$$
\begin{aligned}
Z_{\perp}(k)= & 2 j Z_{0} \int_{-\infty}^{\infty} d q q^{2}|A(q)|^{2}\left[\frac { 1 } { u ^ { 2 } } \left(-\frac{u J_{1}{ }^{\prime}(u)}{J_{1}(u)}\right.\right. \\
& \left.\left.+\frac{J_{1}(u)}{u J_{1}{ }^{\prime}(u)}\right)\right]_{u=a_{0} \sqrt{k^{2}-(k-q)^{2}}}
\end{aligned}
$$

Expanding the Bessel functions in terms of their zeros, he also wrote the impedance in the following form: 


$$
\begin{aligned}
Z_{\perp}(k)= & \frac{-2 j Z_{0}}{a_{0}^{2}} \int_{-\infty}^{\infty} d q q^{2}|A(q)|^{2} \\
& \times \sum_{n=1}^{\infty}\left\{\frac{1}{\left(\nu_{n}^{2}-1\right)\left[k q-\frac{q^{2}}{2}-\frac{\nu_{n}^{2}}{2 a_{0}^{2}}\right]}+\frac{1}{k q-\frac{q^{2}}{2}-\frac{\mu_{n}^{2}}{2 a_{0}^{2}}}\right\},
\end{aligned}
$$

where $J_{1}\left(\mu_{n}\right)=0$ and $J_{1}^{\prime}\left(\nu_{n}\right)=0$. If $a(z)$ varies on the length scale $\ell$, then $A(q)$ will be small for $q \gg 1 / \ell$. In this case, we see that Eq. (2.3) reduces to Yokoya's approximation (1.1) when $a_{0} \ll \ell$ and $k a_{0}^{2} \ll \ell$. Equation (2.3) holds unambiguously for $0 \leq k<\mu_{1} / a_{0}$, and the impedance is seen to be positive imaginary in this region.

In order to find a useful expression for the impedance for $k>\mu_{1} / a_{0}$, we rewrite Eq. (2.3) in a form suggested by the work of Warnock [10]. Using

$$
q^{2}|A(q)|^{2}=\int \frac{d z_{1}}{2 \pi} \frac{d z_{2}}{2 \pi} e^{j q\left(z_{1}-z_{2}\right)} S^{\prime}\left(z_{1}\right) S^{\prime}\left(z_{2}\right),
$$

Eq. (2.3) implies

$$
Z_{\perp}(k)=\frac{j Z_{0}}{\pi a_{0}^{2}} \sum_{n=1}^{\infty}\left\{\frac{F\left(k_{n}{ }^{\prime}, k\right)}{\left(\nu_{n}^{2}-1\right)\left(-j k_{n}{ }^{\prime}\right)}+\frac{F\left(k_{n}, k\right)}{\left(-j k_{n}\right)}\right\},
$$

where

$$
\begin{aligned}
k_{n} & =\sqrt{k^{2}-\frac{\mu_{n}^{2}}{a_{0}^{2}}} \text { and } k_{n}{ }^{\prime}=\sqrt{k^{2}-\frac{\nu_{n}^{2}}{a_{0}^{2}}} \\
F(p, k) & =\int_{-\infty}^{\infty} d z_{1} \int_{-\infty}^{\infty} d z_{2} S^{\prime}\left(z_{1}\right) S^{\prime}\left(z_{2}\right) e^{j k\left(z_{1}-z_{2}\right)} e^{j p\left|z_{1}-z_{2}\right|} \\
& =H(p, k)+H(p,-k), \\
H(p, k) & =\int_{-\infty}^{\infty} d z_{1} \int_{-\infty}^{z_{1}} d z_{2} S^{\prime}\left(z_{1}\right) S^{\prime}\left(z_{2}\right) e^{j(p+k)\left(z_{1}-z_{2}\right)}
\end{aligned}
$$

Since $H^{*}(p, k)=H\left(-p^{*},-k\right)$, it follows that $F(p, k)=$ $H(p, k)+H^{*}\left(-p^{*}, k\right)$. Hence, if $k$ is small enough to assure that all $k_{n}$ and $k_{n}{ }^{\prime}$ are pure imaginary, then $Z_{\perp}(k)$ is pure inductive. The square roots in Eq. (2.7) are chosen to be positive imaginary for negative real argument and negative real for positive real argument. This assures that both $\operatorname{Im} Z_{\perp}(k)$ and $\operatorname{Re} Z_{\perp}(k)$ are positive for positive real $k$. The impedance for negative $k$ can be determined from $Z_{\perp}(-k)=-Z_{\perp}^{*}(k)$. Equation (2.6) is a useful representation for numerical evaluation.

Let us now consider an explicit example [10] of a wall perturbation:

$$
\begin{array}{rlrl}
S(z) & =-\varepsilon \cos \left(\frac{\pi z}{g}\right) & & -g \leq z \leq g \\
& =\varepsilon & |z| \geq g .
\end{array}
$$

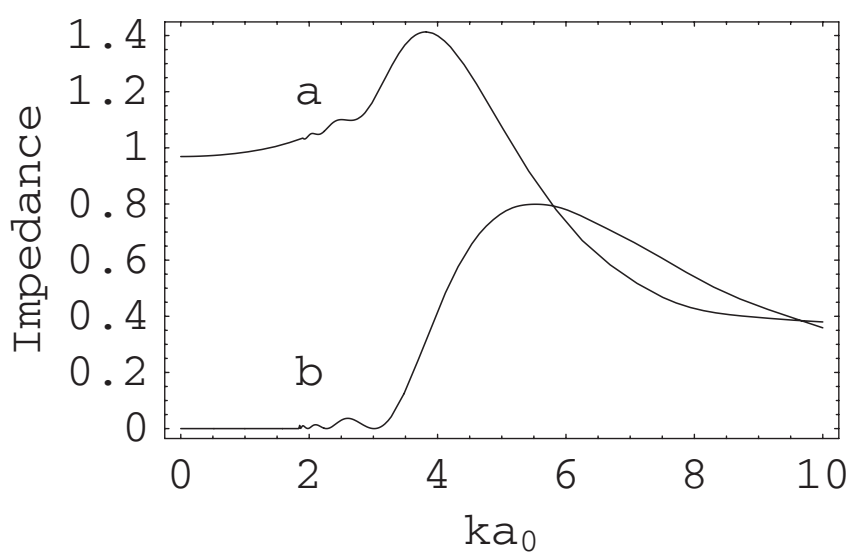

FIG. 1. The imaginary (a) and real (b) parts of the transverse impedance versus $k a_{0}$, for $g / a_{0}=10$, normalized by the Yokoya value $Z_{0}(\pi \varepsilon)^{2} /(2 \pi g)$. Quantities plotted are dimensionless.

In this case,

$$
A(q)=-\frac{\pi \varepsilon \sin q g}{q\left(\pi^{2}-q^{2} g^{2}\right)}+\varepsilon \delta(q)
$$

where $\delta(q)$ is the Dirac delta function and

$$
\begin{aligned}
H(g, p, k)= & (\pi \varepsilon)^{2}\left[\frac{j g(k+p)}{g^{2}(k+p)^{2}-\pi^{2}}\right. \\
& \left.+\frac{\pi^{2}\left[1-e^{2 j g(k+p)}\right]}{\left[g^{2}(k+p)^{2}-\pi^{2}\right]^{2}}\right] .
\end{aligned}
$$

For $g / a_{0}=10$, the real and imaginary parts of the transverse impedance are plotted in Fig. 1. When $k$ is in the vicinity of the roots $k \sim \mu_{n} / a_{0}$ and $k \sim \nu_{n} / a_{0}$ an apparent singular behavior is observed. As discussed by Warnock [10] in his paper on the longitudinal impedance, it is likely that this is an artifact of the perturbation theory. In Fig. 1, the singular behavior that occurs over a narrow range of $k$ is not apparent.

\section{FLAT CHAMBER}

To calculate the vertical impedance, we begin by considering the electromagnetic field generated by a vertical dipole distribution at $x=y=0$ situated between perfectly conducting parallel plates separated by half-distance $b_{0}$. Assuming the electrons are highly relativistic $(\gamma \rightarrow \infty)$, the electromagnetic field can be written in the form (see Appendix A)

$$
\begin{gathered}
B_{y}^{(0)}=E_{x}^{(0)}=-\frac{\partial \phi}{\partial x}, \\
-B_{x}^{(0)}=E_{y}^{(0)}=-\frac{\partial \phi}{\partial y}, \\
B_{z}^{(0)}=E_{z}^{(0)}=0
\end{gathered}
$$


where all fields are multiplied by the factor $e^{j \omega t-j k z}$. The potential $\phi$ is the sum of the contribution of the dipole at the origin (with dipole moment $\lambda=2 e \Delta$ ) and the images induced by the parallel plates,

$$
\phi=\lambda \sum_{n=-\infty}^{\infty} \frac{y+2 n b_{0}}{x^{2}+\left(y+2 n b_{0}\right)^{2}} .
$$

This can be rewritten in a form more useful for calculation,

$$
\phi=\lambda \frac{\pi}{2 b_{0}} \frac{\sin \left(\pi y / b_{0}\right)}{\cosh \left(\pi x / b_{0}\right)-\cos \left(\pi y / b_{0}\right)} .
$$

The vertical impedance is expressed in terms of the fields via $\left[Z_{0}=4 \pi / c\right]$

$$
Z_{y}(k)=\frac{j Z_{0}}{4 \pi e \Delta} \int_{-\infty}^{\infty} d z\left(E_{y}+B_{x}\right) .
$$

It follows from Eq. (3.2) that the impedance vanishes for constant separation between the plates.

Following the approach of Ref. [9], we consider a periodic variation of the half-gap given by the Fourier expansion

$$
b(z)=b_{0}\left[1+\sum_{p=-\infty}^{\infty} \tilde{C}_{p} e^{j(2 \pi p / L) z}\right] .
$$

The boundary conditions for a perfectly conducting wall are that both parallel components of the electric field and the normal component of the magnetic field vanish at $y=$ $\pm b(z)$. The perturbation technique [9] involves expanding the fields in orders of the quantity $\tilde{C}_{p}$ defined in Eq. (3.7), e.g.,

$$
E_{y}=E_{y}^{(0)}+E_{y}^{(1)}+E_{y}^{(2)} .
$$

The zeroth-order approximation consists of the fields that would be found between parallel plates with constant separation, $2 b_{0}$. Order-by-order, the boundary conditions at $y= \pm b(z)$ are replaced [7] by appropriate boundary conditions at $y= \pm b_{0}$, as described in Appendix B.

To carry out the perturbation expansion, we first determine the zeroth-order vertical electric field on the plates,

$$
\begin{aligned}
E_{y}^{(0)}\left(x, b_{0}\right) & =\lambda \frac{\pi^{2}}{2 b_{0}^{2}} \frac{1}{1+\cosh \left(\pi x / b_{0}\right)} \\
& =\lambda \int_{0}^{\infty} d \mu \frac{\mu \cos \mu x}{\sinh \mu b_{0}},
\end{aligned}
$$

and the derivative with respect to the vertical coordinate of the vertical magnetic field on the plates,

$$
\begin{aligned}
\frac{\partial B_{y}^{(0)}}{\partial y}\left(x, b_{0}\right) & =\frac{\partial E_{x}^{(0)}}{\partial y}\left(x, b_{0}\right)=\frac{\partial E_{y}^{(0)}}{\partial x}\left(x, b_{0}\right) \\
& =-\lambda \int_{0}^{\infty} d \mu \frac{\mu^{2} \sin \mu x}{\sinh \mu b_{0}} .
\end{aligned}
$$

The higher-order fields can be expressed in the form,

$$
\begin{aligned}
f(x, y, z, t)= & \int_{-\infty}^{\infty} d k e^{j k(c t-z)} \sum_{p=-\infty}^{\infty} e^{j(2 \pi p / L) z} \\
& \times \int_{-\infty}^{\infty} d \mu \tilde{f}(y, k, p, \mu)\left\{\begin{array}{c}
\cos \mu x \\
\text { or } \\
\sin \mu x
\end{array}\right\},
\end{aligned}
$$

where

$$
E_{y}, E_{z}, B_{x} \propto \cos \mu x, \quad E_{x}, B_{y}, B_{z} \propto \sin \mu x .
$$

From the wave equation, it follows that

$$
\left(-\mu^{2}+\frac{\partial^{2}}{\partial y^{2}}-k_{p}^{2}+k^{2}\right) \tilde{f}=0
$$

with

$$
k_{p}=\frac{2 \pi p}{L}-k
$$

The curl equations (Appendix A) can then be used to show that

$$
B_{y}, E_{x}, E_{z} \propto \sin \nu_{p} y, \quad B_{x}, B_{z}, E_{y} \propto \cos \nu_{p} y,
$$

where

$$
\nu_{p}^{2}=\alpha_{p}^{2}-\mu^{2}, \quad \alpha_{p}^{2}=k^{2}-k_{p}^{2} .
$$

We now use the boundary conditions at $y= \pm b_{0}$ to express the first-order fields in terms of the zeroth-order fields (see Appendix B), and find

$$
\begin{aligned}
\tilde{E}_{z}^{(1)}\left(b_{0}, k, p, \mu\right) & =-\tilde{E}_{y}^{(0)}\left(b_{0}, k, 0, \mu\right) b_{0} \tilde{C}_{p} \frac{2 \pi p j}{L} \\
& =-b_{0} \tilde{C}_{p} \frac{2 \pi p j}{L} \lambda \frac{\mu}{\sinh \mu b_{0}}, \\
\tilde{B}_{y}^{(1)}\left(b_{0}, k, p, \mu\right) & =-\frac{\partial \tilde{B}_{y}^{(0)}}{\partial y}\left(b_{0}, k, 0, \mu\right) b_{0} \tilde{C}_{p} \\
& =b_{0} \tilde{C}_{p} \lambda \frac{\mu^{2}}{\sinh \mu b_{0}} \\
\tilde{E}_{x}^{(1)}\left(b_{0}, k, p, \mu\right) & =-\frac{\partial \tilde{E}_{x}^{(0)}}{\partial y}\left(b_{0}, k, 0, \mu\right) b_{0} \tilde{C}_{p} \\
& =b_{0} \tilde{C}_{p} \lambda \frac{\mu^{2}}{\sinh \mu b_{0}}=\tilde{B}_{y}^{(1)}\left(b_{0}, k, p, \mu\right) .
\end{aligned}
$$

From the curl equations, one can show

$$
\frac{\partial \tilde{B}_{z}^{(1)}}{\partial y}=j k_{p} \tilde{B}_{y}^{(1)}+j k \tilde{E}_{x}^{(1)}=j\left(k_{p}+k\right) \tilde{B}_{y}^{(1)}=j \frac{2 \pi p}{L} \tilde{B}_{y}^{(1)} .
$$

It then follows from Eqs. (3.15) and (3.17) that 


$$
\tilde{E}_{z}^{(1)}(y, k, p, \mu)=D_{p}(\mu) \frac{\sin \nu_{p} y}{\sin \nu_{p} b_{0}}
$$

and from Eqs. (3.15) and (3.20) that

$$
\tilde{B}_{z}^{(1)}(y, k, p, \mu)=\mu D_{p}(\mu) \frac{\cos \nu_{p} y}{\nu_{p} \sin \nu_{p} b_{0}},
$$

where we have defined

$$
D_{p}(\mu)=-\lambda b_{0} \tilde{C}_{p} \frac{2 \pi p j}{L} \frac{\mu}{\sinh \mu b_{0}} .
$$

Having determined the first-order fields, we can now use the second-order boundary condition to show that (Appendix B)

$$
\begin{aligned}
\tilde{E}_{z}^{(2)}\left(b_{0}, k, 0, \mu\right)= & -\sum_{q}\left(\frac{\partial \tilde{E}_{z}^{(1)}}{\partial y}\right)_{q} b_{0} \tilde{C}_{-q} \\
& -\sum_{q}\left(\tilde{E}_{y}^{(1)}\right)_{q} b_{0} \tilde{C}_{-q} \frac{2 \pi j(-q)}{L} .
\end{aligned}
$$

The curl equations imply (see Appendix A)

$$
\alpha_{p}^{2} \tilde{E}_{y}^{(1)}=j k_{p} \frac{\partial \tilde{E}_{z}^{(1)}}{\partial y}+j k \frac{\partial \tilde{B}_{z}^{(1)}}{\partial x} .
$$

Inserting this into Eq. (3.24), we find

$$
\begin{aligned}
\tilde{E}_{z}^{(2)}\left(b_{0}, k, 0, \mu\right)= & -\sum_{q} b_{0} \tilde{C}_{-q} \frac{\alpha_{q}^{2}+\frac{2 \pi q}{L} k_{q}}{\alpha_{q}^{2}}\left(\frac{\partial \tilde{E}_{z}^{(1)}}{\partial y}\right)_{q} \\
& -\sum_{q} b_{0} \tilde{C}_{-q} \frac{\frac{2 \pi q}{L} k}{\alpha_{q}^{2}}\left(\frac{\partial \tilde{B}_{z}}{\partial x}\right)_{q}
\end{aligned}
$$

Equations (3.21), (3.22), and (3.23) together with (3.26) imply

$$
\begin{aligned}
\tilde{E}_{z}^{(2)}\left(b_{0}, k, 0, \mu\right)= & j \lambda k b_{0}^{2} \frac{\mu}{\sinh \mu b_{0}} \sum_{q}\left|\tilde{C}_{q}\right|^{2}\left(\frac{2 \pi q}{L}\right)^{2} \\
& \times \frac{\cos \nu_{q} b_{0}}{\nu_{q} \sin \nu_{q} b_{0}} .
\end{aligned}
$$

The vertical impedance per period is given by

$$
Z_{y}(k)=\frac{j Z_{0}}{4 \pi e \Delta} \int_{-L / 2}^{L / 2} d z\left(E_{y}+B_{x}\right) .
$$

From the curl equations (Appendix A), we see that

$$
\frac{\partial \tilde{E}_{z}}{\partial y}=-j k\left(\tilde{E}_{y}+\tilde{B}_{x}\right) \quad \text { for } p=0
$$

Using this in Eq. (3.28), yields

$$
Z_{y}(k)=\frac{j Z_{0}}{4 \pi e \Delta} L \frac{j}{k} \frac{\partial \hat{E}_{z}}{\partial y}(y=0, k, p=0, x=0),
$$

where

$$
\begin{aligned}
\hat{E}_{z}(y, k, p & =0, x)=\int_{-\infty}^{\infty} d \mu \frac{\sinh \mu y}{\sinh \mu b_{0}} \tilde{E}_{z}\left(b_{0}, k, p\right. \\
& =0, \mu) \cos \mu x .
\end{aligned}
$$

From Eqs. (3.21), (3.30), and (3.31), it follows that there is no first-order contribution to the transverse impedance. Using Eqs. (3.27), (3.30), and (3.31), the second-order contribution is found to be

$$
\begin{aligned}
Z_{y}(k)= & \frac{-j Z_{0}}{2 \pi} L b_{0}^{2} \int_{-\infty}^{\infty} d \mu\left(\frac{\mu}{\sinh \mu b_{0}}\right)^{2} \sum_{q}\left|\tilde{C}_{q}\right|^{2}\left(\frac{2 \pi q}{L}\right)^{2} \\
& \times \frac{\cos \nu_{q} b_{0}}{\nu_{q} \sin \nu_{q} b_{0}} .
\end{aligned}
$$

Now let us consider the limit of long period length, $L \rightarrow \infty$. We define

$$
Q=\frac{2 \pi q}{L} \quad \text { and } \quad \nu^{2}=2 Q k-Q^{2}-\mu^{2},
$$

and introduce $A(q)$ via

$$
\frac{b(z)-b_{0}}{b_{0}}=\sum_{q} \frac{L \Delta Q}{2 \pi} \tilde{C}(Q) e^{j Q z} \rightarrow \int_{-\infty}^{\infty} d Q A(Q) e^{j Q z} .
$$

It then follows from Eq. (3.32) that

$$
\begin{aligned}
Z_{y}(k)= & -j Z_{0} b_{0}^{2} \int_{-\infty}^{\infty} d \mu\left(\frac{\mu}{\sinh \mu b_{0}}\right)^{2} \\
& \times \int_{-\infty}^{\infty} d Q|A(Q)|^{2} Q^{2} \frac{\cot \left[\nu(Q, \mu, k) b_{0}\right]}{\nu(Q, \mu, k)} .
\end{aligned}
$$

Exchanging the order of integration, it is seen that

$$
Z_{y}(k)=j Z_{0} \int_{-\infty}^{\infty} d Q|A(Q)|^{2} Q^{2} f\left(Q b_{0}, k b_{0}\right),
$$

where

$$
f(\eta, \kappa)=\int_{-\infty}^{\infty} d \xi\left(\frac{\xi}{\sinh \xi}\right)^{2} \frac{\operatorname{coth} \sqrt{\eta^{2}+\xi^{2}-2 \eta \kappa}}{\sqrt{\eta^{2}+\xi^{2}-2 \eta \kappa}} .
$$

\section{DISCUSSION OF RESULTS}

Let us first consider the impedance at zero frequency,

$$
Z_{y}(0)=j Z_{0} \int_{-\infty}^{\infty} d Q|A(Q)|^{2} Q^{2} f\left(Q b_{0}, 0\right) .
$$

Suppose $b(z)$ varies on the axial length scale $\ell \gg b_{0}$, then $A(Q)$ will be small for $Q \gg 1 / \ell$. In this case we can use the small argument approximation (see Appendix C), 


$$
f(\eta, 0) \cong \frac{\pi}{|\eta|}-1 \quad(\eta \rightarrow 0) .
$$

Employing Eq. (4.2) in (4.1), we find

$$
Z_{y}^{\text {flat }}(0) \cong \frac{j \pi Z_{0}}{b_{0}} \int_{-\infty}^{\infty} d Q|A(Q)|^{2}|Q| .
$$

This can be compared with Yokoya's [2] result (1.1) for the circular case,

$$
Z_{\perp}^{\text {round }}(0)=j Z_{0} \int_{-\infty}^{\infty} d Q|A(Q)|^{2} Q^{2}=j Z_{0} \int_{-\infty}^{\infty} \frac{d z}{2 \pi} S^{\prime}(z)^{2} .
$$

For a slow taper, the flat impedance is larger than the round impedance by a factor $\sim \pi \ell / b_{0}$. Equation (4.3) indicates why Stupakov's perturbation approach is not applicable for plates of infinite width. In this case the impedance is not expressible simply in terms of the derivative of $S(z)$.

To compare with Stupakov's [4] result for a flat taper of finite half-width $w$, let us assume $b_{0} \ll w \ll \ell$ and introduce a low wave number cutoff, $\alpha b_{0} / w$, in the integral defining $f(\eta, 0)$ in Eq. (3.37),

$$
f_{w}(\eta, 0) \equiv 2 \int_{\alpha b_{0} / w}^{\infty} d \xi \frac{\xi^{2}}{\sinh ^{2} \xi} \frac{\cosh \sqrt{\xi^{2}+\eta^{2}}}{\sqrt{\xi^{2}+\eta^{2}} \sinh \sqrt{\xi^{2}+\eta^{2}}}
$$

where $\alpha$ is an unknown constant. Since $\alpha b_{0} / w$ is small, it follows from the analysis of Appendix $\mathrm{C}$ that

$$
f_{w}(0,0) \cong \frac{2 w}{\alpha b_{0}}-1
$$

so

$$
\begin{aligned}
Z_{y}(0) & \cong j Z_{0} \frac{2 w}{\alpha b_{0}} \int_{-\infty}^{\infty} d Q|A(Q)|^{2} Q^{2} \\
& =j Z_{0} \frac{2 w}{\alpha b_{0}} \int_{-\infty}^{\infty} \frac{d z}{2 \pi} S^{\prime}(z)^{2} .
\end{aligned}
$$

This has the same form as Stupakov's result [4], which we expect to be correct for $b_{0} \ll w \ll g$, and we can get agreement with Stupakov [4] by taking $\alpha=2 / \pi$. In the case $w \gg g \gg b_{0}$, we expect $Z_{y}(0)$ will saturate at the value given in Eq. (4.3).

We can write the impedance in a form analogous to Eq. (2.7) which we derived for circular cross section. We use Eq. (2.5) and the identity.

$$
\begin{aligned}
& \frac{\cos \nu b_{0}}{\nu \sin \nu b_{0}}=\sum_{n=0}^{\infty} \frac{\delta_{n} b_{0}}{\left(\nu b_{0}\right)^{2}-(n \pi)^{2}} \\
& \left(\begin{array}{ll}
\delta_{n}=1 \text { for } n=0 & \\
& \delta_{n}=2 \text { for } n>0
\end{array}\right)
\end{aligned}
$$

in Eq. (3.35) to derive

$$
Z_{y}(k)=\frac{j Z_{0}}{2 \pi b_{0}} \int_{-\infty}^{\infty} d \xi \frac{\xi^{2}}{\sinh ^{2} \xi} \sum_{n=0}^{\infty} \frac{\delta_{n} F\left(k_{n}, k\right)}{2\left(-j k_{n}\right) b_{0}},
$$

where

$$
\begin{gathered}
k_{n} b_{0}=\sqrt{\left(k b_{0}\right)^{2}-\xi^{2}-(n \pi)^{2}} \\
F(p, k)=H(p, k)+H(p,-k) \\
H(p, k)=\int_{-\infty}^{\infty} d z_{1} \int_{-\infty}^{z_{1}} d z_{2} S^{\prime}\left(z_{1}\right) S^{\prime}\left(z_{2}\right) e^{i(p+k)\left(z_{1}-z_{2}\right)} .
\end{gathered}
$$

This form is convenient for numerical evaluation.

Let us again consider the example [Eq. (2.10)]

$$
\begin{aligned}
S(z)=\frac{b(z)-b_{0}}{b_{0}} & =-\varepsilon \cos \frac{\pi z}{g} & & (-g<z<g) \\
& =\varepsilon & & (|z|>g) .
\end{aligned}
$$

In this case,

$$
Q^{2}|A(Q)|^{2}=\frac{\pi^{2} \varepsilon^{2} \sin ^{2}(g Q)}{\left(\pi^{2}-g^{2} Q^{2}\right)^{2}},
$$

so Eq. (3.36) becomes

$$
Z_{y}(k)=\frac{2 j Z_{0}}{b_{0}}(\pi \varepsilon)^{2} \int_{0}^{\infty} d \eta \frac{\sin ^{2}\left(g \eta / b_{0}\right)}{\left(\pi^{2}-g^{2} \eta^{2} / b_{0}^{2}\right)^{2}} f\left(\eta, k b_{0}\right) .
$$

For $b_{0} \ll g$, we can use the small argument approximation (4.2) for $f(\eta, 0)$ to show that

$$
Z_{y}(0) \cong \frac{2 j Z_{0}}{b_{0}}(\pi \varepsilon)^{2}\left[0.111-\frac{b_{0}}{4 \pi g}\right] .
$$

More generally, we expect Eq. (4.16) will hold under the condition, $b_{0} \ll g \ll w$.

For $S(z)$ as specified in Eq. (4.13), the function $H$ defined in Eq. (4.12) is

$$
\begin{aligned}
H(g, p, k)= & (\pi \varepsilon)^{2}\left[\frac{j g(k+p)}{g^{2}(k+p)^{2}-\pi^{2}}\right. \\
& \left.+\frac{\pi^{2}\left[1-e^{2 j g(k+p)}\right]}{\left[g^{2}(k+p)^{2}-\pi^{2}\right]^{2}}\right] .
\end{aligned}
$$

Using Eqs. (4.11) and (4.17) in the representation of the impedance given in Eq. (4.9), we numerically evaluate the impedance as a function of $k$. The results are plotted in Fig. 2. To obtain the plots shown in Fig. 2, we have given $k$ a small imaginary part $\sim 10^{-3}$ in order to smooth out some singular behavior occurring when the quantity in the square root in Eq. (4.10) vanishes. As discussed by Warnock [10] for the longitudinal impedance, we believe this singular behavior is an artifact of perturbation theory. 

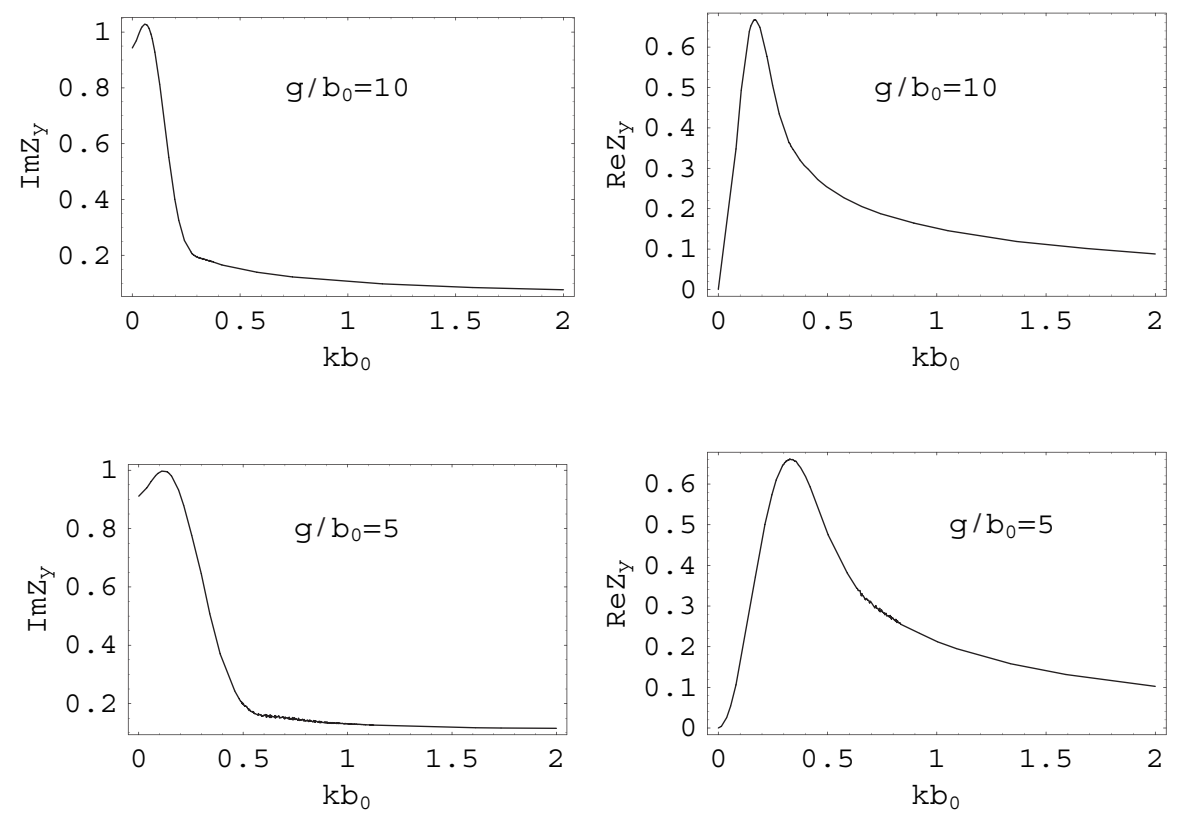

FIG. 2. The real and imaginary parts of the vertical impedance for the flat taper of infinite width $\left(\mathrm{g} / b_{0}=10\right.$ and 5$)$. The impedance is normalized by the approximate value at zero frequency, $\operatorname{Im} Z_{y}(0) \cong\left(0.222 Z_{0} / b_{0}\right)(\pi \varepsilon)^{2}$. Quantities plotted are dimensionless.

The value of the $\operatorname{Im} Z_{y}$ is seen to fall from its maximum value over a wave number range of $\Delta k \sim 1 / g$. The "trapped mode" resonance observed in simulations by Blednykh and Wang [11] is not found within the approximations we have made.

\section{CONCLUDING REMARKS}

The work of Stupakov $[4,5]$ demonstrated that the impedance of flat tapered structures can be significantly larger than that of tapers with circular cross section. In fact, he showed that in the regime $b_{0} \ll w \ll \ell$ and $k w^{2} \ll \ell, \operatorname{Im} Z_{y}$ is expected to increase linearly with the width $w$. His analysis does not cover the regime of larger widths for which the linear increase must saturate. In this paper, we take a first step toward providing an analytic description of wide tapers by determining the impedance of a flat taper of infinite width using the boundary perturbation method employed successfully by Cooper, Krinsky, and Morton [9] in the case of axially symmetric structures. The validity of this approximation requires not only that the taper be slow, but also that the total gap variation be restricted. In future work, it is hoped that the method of Stupakov can be extended to cover wide tapers whose width $w$ is on the order of or larger than the taper length $\ell$. Such a result would not restrict gap variation of the total taper.

Let us note that the impedance at zero frequency found in Eq. (4.3) is not easily expressible in the form expected from Stupakov's perturbation theory. This indicates that in order to apply Stupakov's approach [4,5] a significant modification of the method of solution will be required.

\section{ACKNOWLEDGMENTS}

I wish to thank Gennady Stupakov for a clarifying discussion on his work [3-5]. I have also benefited from discussions with Boris Podobedov and Alexei Blednykh on numerical results they have obtained from computer simulations of tapered structures. This work was supported by Department of Energy Contract No. DE-AC0298CH10886.

\section{APPENDIX A: CURL EQUATIONS}

The Maxwell curl equations are

$$
\begin{array}{ll}
\frac{\partial B_{y}}{\partial x}-\frac{\partial B_{x}}{\partial y}=j k E_{z} & \frac{\partial B_{x}}{\partial z}-\frac{\partial B_{z}}{\partial x}=j k E_{y} \\
\frac{\partial B_{z}}{\partial y}-\frac{\partial B_{y}}{\partial z}=j k E_{x} & \frac{\partial E_{y}}{\partial x}-\frac{\partial E_{x}}{\partial y}=-j k B_{z} \\
\frac{\partial E_{x}}{\partial z}-\frac{\partial E_{z}}{\partial x}=-j k B_{y} & \frac{\partial E_{z}}{\partial y}-\frac{\partial E_{y}}{\partial z}=-j k B_{x} .
\end{array}
$$

Using these it follows that

$$
\begin{aligned}
& \frac{\partial^{2} E_{y}}{\partial z^{2}}=\frac{\partial^{2} E_{y}}{\partial z \partial y}+j k \frac{\partial B_{x}}{\partial z}=\frac{\partial^{2} E_{y}}{\partial z \partial y}+j k\left(\frac{\partial B_{z}}{\partial x}+j k E_{y}\right) \\
& \left(\frac{\partial^{2}}{\partial z^{2}}+k^{2}\right) E_{y}=\frac{\partial^{2} E_{y}}{\partial z \partial y}+j k \frac{\partial B_{z}}{\partial x} \\
& \alpha_{p}^{2} E_{y}=j k_{p} \frac{\partial E_{z}}{\partial y}+j k \frac{\partial B_{z}}{\partial x}
\end{aligned}
$$

where 


$$
\alpha_{p}^{2}=k^{2}-k_{p}^{2} \quad k_{p}=\frac{2 \pi p}{L}-k .
$$

When $E_{z}=0, B_{z}=0, k_{p}=-k(p=0)$, the curl equations reduce to

$$
\begin{aligned}
& \frac{\partial B_{y}}{\partial x}-\frac{\partial B_{x}}{\partial y}=Z_{0} J_{z} \quad\left(Z_{0}=\frac{4 \pi}{c}\right) \\
& -j k B_{x}=j k E_{y} \\
& j k B_{y}=j k E_{x} \\
& \frac{\partial E_{y}}{\partial x}-\frac{\partial E_{x}}{\partial y}=0 \\
& -j k E_{x}=-j k B_{y} \\
& j k E_{y}=-j k B_{x} .
\end{aligned}
$$

It is now easily seen that in this case the fields are expressible in terms of a scalar potential via

$$
\begin{aligned}
& -B_{x}=E_{y}=-\frac{\partial \phi}{\partial y} \\
& B_{y}=E_{x}=-\frac{\partial \phi}{\partial x} \\
& \frac{\partial^{2} \phi}{\partial x^{2}}+\frac{\partial^{2} \phi}{\partial y^{2}}=-Z_{0} J_{z} .
\end{aligned}
$$

\section{APPENDIX B: BOUNDARY CONDITIONS}

The boundary conditions for the perfectly conducting wall are that both the parallel components of the electric field and the normal component of the magnetic field vanish at $y= \pm b(z)$.

$$
\begin{gathered}
{\left[E_{z}+\frac{d b}{d z} E_{y}\right]_{y= \pm b(z)}=0,} \\
{\left[E_{x}\right]_{y= \pm b(z)}=0,} \\
{\left[B_{y}-\frac{d b}{d z} B_{z}\right]_{y= \pm b(z)}=0 .}
\end{gathered}
$$

We can expand the field components in a Taylor series such as

$$
\begin{aligned}
{\left[E_{z}\right]_{b(z)}=} & {\left[E_{z}\right]_{b_{0}}+\left[\frac{\partial E_{z}}{\partial y}\right]_{b_{0}}\left[b(z)-b_{0}\right]+\frac{1}{2}\left[\frac{\partial^{2} E_{z}}{\partial y^{2}}\right]_{b_{0}} } \\
& \times\left[b(z)-b_{0}\right]^{2}
\end{aligned}
$$

For example combining Eqs. (B1) and (B4), we obtain the constraint at $y=b_{0}$,

$$
\begin{aligned}
{\left[E_{z}\right]_{b_{0}}=} & -\left[\frac{\partial E_{z}}{\partial y}\right]_{b_{0}}\left(b-b_{0}\right)-\left[\frac{\partial^{2} E_{z}}{\partial y^{2}}\right]_{b_{0}} \frac{\left(b-b_{0}\right)^{2}}{2} \\
& -\left[E_{y}\right]_{b_{0}} \frac{d b}{d z}-\left[\frac{\partial E_{y}}{\partial y}\right]_{b_{0}}\left(b-b_{0}\right) \frac{d b}{d z} .
\end{aligned}
$$

Introducing the Fourier transform as described in Eq. (3.12), the constraint in (B5) becomes (all quantities are evaluated at $y=b_{0}$ )

$$
\begin{aligned}
\left(\tilde{E}_{z}\right)_{p}= & -\sum_{q}\left(\frac{\partial \tilde{E}_{z}}{\partial y}\right)_{q} b_{0} \tilde{C}_{p-q} \\
& -\sum_{q}\left(\tilde{E}_{y}\right)_{q} b_{0} \tilde{C}_{p-q} \frac{2 \pi(p-q) j}{L} \\
& -\frac{1}{2} \sum_{q, n}\left(\frac{\partial^{2} \tilde{E}_{z}}{\partial y^{2}}\right)_{q} b_{0} \tilde{C}_{p-q-n} b_{0} \tilde{C}_{n} \\
& -\sum_{q}\left(\frac{\partial \tilde{E}_{y}}{\partial y}\right)_{q} b_{0} \tilde{C}_{p-q-n} b_{0} \tilde{C}_{n} \frac{2 \pi n j}{L}
\end{aligned}
$$

The field components are now expanded in orders of $\tilde{C}_{p}$, e.g.

$$
\tilde{E}_{y}=\tilde{E}_{y}^{(0)}+\tilde{E}_{y}^{(1)}+\tilde{E}_{y}^{(2)}
$$

where $\tilde{E}_{y}^{(0)}$ is the solution for a constant radius $b_{0}$, the term $\tilde{E}_{y}^{(1)}$ is linear and the term $\tilde{E}_{y}^{(2)}$ quadratic in the coefficients $\tilde{C}_{p}$.

We recall that $E_{z}^{(0)}=B_{z}^{(0)}=0$, and that the zeroth-order field components, e.g., $\left(\tilde{E}_{y}^{(0)}\right)_{p}$, are only nonvanishing for $p=0$. Then to first order, we obtain the constraints

$$
\begin{gathered}
\tilde{E}_{z}^{(1)}\left(b_{0}, k, p, \mu\right)=-\tilde{E}_{y}^{(0)}\left(b_{0}, k, 0, \mu\right) b_{0} \tilde{C}_{p} \frac{2 \pi p j}{L}, \\
\tilde{B}_{y}^{(1)}\left(b_{0}, k, p, \mu\right)=-\frac{\partial \tilde{B}_{y}^{(0)}}{\partial y}\left(b_{0}, k, 0, \mu\right) b_{0} \tilde{C}_{p}, \\
\tilde{E}_{x}^{(1)}\left(b_{0}, k, p, \mu\right)=-\frac{\partial \tilde{E}_{x}^{(0)}}{\partial y}\left(b_{0}, k, 0, \mu\right) b_{0} \tilde{C}_{p} .
\end{gathered}
$$

We can also derive the second-order constraint,

$$
\begin{aligned}
\tilde{E}_{z}^{(2)}\left(b_{0}, k, 0, \mu\right)= & -\sum_{q}\left(\frac{\partial \tilde{E}_{z}^{(1)}}{\partial y}\right)_{q} b_{0} \tilde{C}_{-q} \\
& -\sum_{q}\left(\tilde{E}_{y}^{(1)}\right)_{q} b_{0} \tilde{C}_{-q} \frac{2 \pi j(-q)}{L} \\
& -\sum_{n}\left(\frac{\partial \tilde{E}_{z}^{(0)}}{\partial y}\right)_{0} b_{0} \tilde{C}_{-n} b_{0} \tilde{C}_{n} \frac{2 \pi n j}{L},
\end{aligned}
$$

where the last term vanishes since the summand is odd in $n$. 
APPENDIX C: DERIVATION OF EQ. (4.2)

$$
\begin{gathered}
f(\eta)=2 \int_{0}^{\infty} d \xi \frac{\xi^{2}}{\sinh ^{2} \xi} \frac{\cosh \sqrt{\xi^{2}+\eta^{2}}}{\sqrt{\xi^{2}+\eta^{2}} \sinh \sqrt{\xi^{2}+\eta^{2}}} \\
\eta \rightarrow 0 \\
f(\eta) \approx 2 \int_{0}^{\varepsilon} \frac{d \xi}{\xi^{2}+\eta^{2}}+2 \int_{\varepsilon}^{\infty} d \xi \frac{\xi \cosh \xi}{\sinh ^{3} \xi} \\
=\frac{2}{\eta} \tan ^{-1} \frac{\varepsilon}{\eta}+(-1+\operatorname{coth} \varepsilon)(1+\varepsilon+\varepsilon \operatorname{coth} \varepsilon)
\end{gathered}
$$

consider $|\eta| \ll \varepsilon \ll 1$

$$
\begin{gathered}
f(\eta) \approx \frac{2}{|\eta|}\left(\frac{\pi}{2}-\frac{|\eta|}{\varepsilon}\right)+\left(-1+\frac{1}{\varepsilon}+\frac{\varepsilon}{3}\right)\left(1+\varepsilon+1+\frac{\varepsilon^{2}}{3}\right) \\
\approx \frac{\pi}{|\eta|}-\frac{2}{\varepsilon}+\left(-2+\frac{2}{\varepsilon}+1\right) \\
f(\eta) \approx \frac{\pi}{|\eta|}-1
\end{gathered}
$$

[1] K. Bane and S. Krinsky, in Proceedings of the Particle Accelerator Conference, Washington DC, 1993 (IEEE, Piscataway, NJ, 1993), p. 3375.

[2] K. Yokoya, Report No. CERN SL/90-88 (AP), 1990.

[3] G. V. Stupakov, Part. Accel. 56, 83 (1995).

[4] G. V. Stupakov, Report No. SLAC-PUB-7167, 1996.

[5] G. V. Stupakov, Report No. SLAC-PUB-8857.

[6] B. Podobedov and S. Krinsky, in Proceedings of the Particle Accelerator Conference, Knoxville, Tennessee, 2005 (IEEE, Piscataway, NJ, 2005).

[7] M. Chatard-Moulin and A. Papiernik, in Proceedings of the Particle Accelerator Conference, San Francisco, 1979 (IEEE, Piscataway, NJ, 1979) [IEEE Trans. Nucl. Sci. 26, 3523 (1979)].

[8] S. Krinsky, in Proceedings of the XIth International Conference on High Energy Accelerators, Geneva, Switzerland, 1980 (Birkhauser-Verlag, Basel, 1980), p. 576.

[9] R. K. Cooper, S. Krinsky, and P. L. Morton, Part. Accel. 12, 1 (1982).

[10] R. L. Warnock, in Proceedings of the Particle Accelerator Conference, Washington, DC, 1993 (Ref. [1]), p. 3378.

[11] A. Blednykh and J.M. Wang (private communication). 